\title{
Boundary conditions in photoacoustic tomography
}

Lihong V. Wang

Lihong V. Wang, "Boundary conditions in photoacoustic tomography," Proc. SPIE 6086, Photons Plus Ultrasound: Imaging and Sensing 2006: The Seventh Conference on Biomedical Thermoacoustics, Optoacoustics, and Acousto-optics, 608611 (6 March 2006); doi: 10.1117/12.647818 


\title{
Boundary conditions in photoacoustic tomography
}

\author{
Lihong V. Wang* \\ Optical Imaging Laboratory \\ Department of Biomedical Engineering \\ Texas A\&M University \\ 3120 TAMU, College Station, Texas 77843-3120
}

\begin{abstract}
Photoacoustic tomography has captured a significant amount of attention recently. Although several commercially available optical imaging modalities, including confocal microscopy, two-photon microscopy, and optical coherence tomography have been highly successful, none of these technologies can penetrate beyond $\sim 1 \mathrm{~mm}$ into scattering biological tissues because all of them are based on ballistic and quasi-ballistic photons. Consequently, until now, there has been a void in high-resolution optical imaging beyond this depth limit. Photoacoustic tomography has filled this void by combining high ultrasonic resolution and high optical contrast in a single modality. Up until now, however, free-space ultrasound propagation has always been assumed in photoacoustic tomography. In this paper, boundary conditions that should be considered in certain imaging configurations are presented and their associated inverse solutions for image reconstruction are provided.
\end{abstract}

Keywords: Photoacoustic tomography, thermoacoustic tomography, image reconstruction, boundary conditions, time reversal

\section{INTRODUCTION}

The motivation for photoacoustic tomography (PAT) is to combine the spatial resolution of ultrasound with the contrast of optical absorption for deep imaging in the diffusion regime. In Table 1, PAT is compared with optical coherence tomography (OCT), diffuse optical tomography (DOT), and ultrasonography (US). At large imaging depths, pure optical imaging suffers from strong light scattering in biological tissues. As a result, the pure optical imaging modalities have either shallow imaging depth, as in OCT, or low spatial resolution, as in DOT. Ultrasound scattering, however, is 2-3 orders of magnitude weaker than optical scattering in biological tissues. As a consequence, pure ultrasonic imaging provides better resolution than pure optical imaging at depths greater than $\sim 1 \mathrm{~mm}$. However, because ultrasound imaging is based on the detection of mechanical properties in biological tissues, it provides only weak contrast for detecting early stage tumors. Moreover, ultrasound is insensitive to biochemical molecules, such as oxygenated and deoxygenated hemoglobins, to which optical absorption is highly sensitive.

PAT overcomes the limitations of both the pure optical and the pure ultrasonic imaging modalities. In this hybrid method, the contrast is related to the optical properties of the sample, yet the resolution is not limited by optical diffusion. The image resolution, as well as the maximum imaging depth, is scaleable with the ultrasonic frequency within the reach of diffuse photons. As the ultrasound frequency and bandwidth is increased, the spatial resolution improves at the expense of maximum imaging depth. For example, photoacoustic signals on the order of $1 \mathrm{MHz}$ can provide a spatial resolution on the order of $1.5 \mathrm{~mm}$ because the speed of sound in soft tissues is $\sim 1.5 \mathrm{~mm} / \mu \mathrm{s}$, whereas photoacoustic signals on the order of $50 \mathrm{MHz}$ can provide a spatial resolution on the order of $30 \mu \mathrm{m}$. In addition, PAT images are devoid of speckle artifacts, which are conspicuously present in OCT and US images.

* Tel: (979) 845-4488; Fax: (979) 845-4450; Email: lwang@tamu.edu; URL: http://oilab.tamu.edu 
Table 1. Comparison of photo-acoustic tomography (PAT), optical coherence tomography (OCT), diffuse optical tomography (DOT), and ultrasonography (US) operated at $6 \mathrm{MHz}$.

\begin{tabular}{|l|l|l|l|l|}
\hline Properties & OCT & DOT & US & PAT \\
\hline Contrast & Good & Excellent & Poor for early cancers & $\begin{array}{l}\text { Excellent } \\
(\approx \text { DOT })\end{array}$ \\
\hline Resolution & $\begin{array}{l}\text { Excellent } \\
(\sim 10 \mu \mathrm{m})\end{array}$ & $\begin{array}{l}\text { Poor } \\
(\sim 5 \mathrm{~mm})\end{array}$ & $\begin{array}{l}\text { Excellent \& scalable } \\
(\sim 150 \mu \mathrm{m})\end{array}$ & $\begin{array}{l}\text { Excellent } \\
(\approx \text { US })\end{array}$ \\
\hline Imaging depth & $\begin{array}{l}\text { Poor } \\
(\sim 1 \mathrm{~mm})\end{array}$ & $\begin{array}{l}\text { Excellent } \\
(\sim 5 \mathrm{~cm})\end{array}$ & $\begin{array}{l}\text { Good \& scalable } \\
(\sim 3 \mathrm{~cm})\end{array}$ & $\begin{array}{l}\text { Good \& scalable } \\
(\approx \text { US })\end{array}$ \\
\hline Speckle artifacts & Strong & None & Strong & None \\
\hline $\begin{array}{l}\text { Scattering } \\
\text { coefficient }\end{array}$ & $\begin{array}{l}\text { Strong } \\
\left(\sim 100 \mathrm{~cm}^{-1}\right)\end{array}$ & $\begin{array}{l}\text { Strong } \\
\left(\sim 100 \mathrm{~cm}^{-1}\right)\end{array}$ & $\begin{array}{l}\text { Weak } \\
\left(\sim 0.3 \mathrm{~cm}^{-1}\right)\end{array}$ & \\
\hline
\end{tabular}

\section{PHOTOACOUSTIC EQUATION}

When a pressure rise is initiated by laser heating, acoustic waves develop and begin to propagate. The acoustic wave generation and propagation in an inviscid medium is described by

$$
\left(\nabla^{2}-\frac{1}{v_{s}^{2}} \frac{\partial^{2}}{\partial t^{2}}\right) p(\vec{r}, t)=-\frac{\beta}{\kappa v_{s}^{2}} \frac{\partial^{2} T}{\partial t^{2}},
$$

where $p(\vec{r}, t)$ is the acoustic pressure at location $\vec{r}$ and time $t ; \kappa$ is the isothermal compressibility $\left[\mathrm{Pa}^{-1}\right.$; the typical value for water or soft tissue is $\left.\sim 5 \times 10^{-5} \operatorname{bar}^{-1}\right]$; and $\beta$ is the thermal coefficient of the volume expansion $\left[\mathrm{K}^{-1} ; \sim 4 \times 10^{-}\right.$ ${ }^{4} \mathrm{~K}^{-1}$ for muscle], respectively. The isothermal compressibility $\kappa$ can be expressed as

$$
\kappa=\frac{C_{P}}{\rho v_{s}^{2} C_{V}}
$$

where $\rho$ is the mass density $\left[\mathrm{kg} / \mathrm{m}^{3} ; \sim 1 \mathrm{~g} / \mathrm{cm}^{3}\right.$ for water and soft tissue]; $v_{s}$ is the speed of sound $[\mathrm{m} / \mathrm{s} ; \sim 1.5 \mathrm{~mm} / \mu \mathrm{s}$ in water]; and $C_{P}$ and $C_{V}\left[\sim 4 \mathrm{~J} \mathrm{~g}^{-1} \mathrm{~K}^{-1}\right.$ for muscle $]$ are the heat capacity $\left[\mathrm{J} \mathrm{kg}^{-1} \mathrm{~K}^{-1}\right]$ at a constant pressure and volume, respectively.

The left-hand side of Eq. (1) describes the wave propagation, while the right-hand side represents the source term.

In thermal confinement, the thermal equation becomes

$\rho C_{V} \frac{\partial T(\vec{r}, t)}{\partial t}=H(\vec{r}, t)$,

where $H(\vec{r}, t)$ is the heating function defined as the thermal energy deposited by the energy source per time and volume. The heating function $H$ is related to the optical energy deposition $Q_{e}$ by

$Q_{e}=\int H d t$

and to the optical fluence rate $\Phi$ by

$H=\mu_{a} \Phi$

if all of the absorbed optical energy is assumed to be converted to heat. Substituting Eq. (3) into Eq. (1), we obtain the following less general photoacoustic equation: 


$$
\left(\nabla^{2}-\frac{1}{v_{s}^{2}} \frac{\partial^{2}}{\partial t^{2}}\right) p(\vec{r}, t)=-\frac{\beta}{C_{P}} \frac{\partial H}{\partial t} .
$$

Here, the source term is expressed as the first derivative of the heating function $H$ with respect to time $t$. This means that constant heating does not produce a pressure wave; only a change in heating does.

Sometimes, velocity potential $\phi_{v}$, which is related to $p(\vec{r}, t)$ by

$$
p=-\rho \frac{\partial \phi_{v}}{\partial t}
$$

is used for convenience so that the time derivative of the heating function can be avoided:

$$
\left(\nabla^{2}-\frac{1}{v_{s}^{2}} \frac{\partial^{2}}{\partial t^{2}}\right) \phi_{v}=\frac{\beta}{\rho C_{P}} H .
$$

\section{FORWARD SOLUTION BY GREEN'S FUNCTION APPROACH}

The general photoacoustic equation shown in Eq. (1) can be solved by the Green's function approach. Green's function is defined as the response to an impulse source term represented by the product of the spatial and the temporal delta functions:

$$
\left(\nabla^{2}-\frac{1}{v_{s}^{2}} \frac{\partial^{2}}{\partial t^{2}}\right) G\left(\vec{r}, t ; \vec{r}^{\prime}, t^{\prime}\right)=-\delta\left(\vec{r}-\vec{r}^{\prime}\right) \delta\left(t-t^{\prime}\right),
$$

where $\vec{r}^{\prime}$ and $t^{\prime}$ denote the source location and time, respectively. In infinite space, where no boundaries are involved, the Green's function can be expressed as

$$
G\left(\vec{r}, t ; \vec{r}^{\prime}, t^{\prime}\right)=\frac{\delta\left(t-t^{\prime}-\frac{\left|\vec{r}-\vec{r}^{\prime}\right|}{v_{s}}\right)}{4 \pi\left|\vec{r}-\vec{r}^{\prime}\right|},
$$

which represents an outgoing spherical wave.

Based on the Green's function approach, the pressure due to an arbitrary source can be represented by

$$
p(\vec{r}, t)=\int_{0}^{t^{+}} d t^{\prime} \int d \vec{r}^{\prime} G\left(\vec{r}, t ; \vec{r}^{\prime}, t^{\prime}\right) \frac{\beta}{\kappa v_{s}^{2}} \frac{\partial^{2} T\left(\vec{r}^{\prime}, t^{\prime}\right)}{\partial t^{\prime 2}}
$$

Substituting Eq. (7) into the above equation leads to

$$
p(\vec{r}, t)=\left.\frac{\beta}{4 \pi \kappa v_{s}^{2}} \int d \vec{r}^{\prime} \frac{1}{\left|\vec{r}-\vec{r}^{\prime}\right|} \frac{\partial^{2} T\left(\vec{r}^{\prime}, t^{\prime}\right)}{\partial t^{\prime 2}}\right|_{t^{\prime}=t-\frac{\left|\vec{r}-\vec{r}^{\prime}\right|}{v_{s}}} \text {. }
$$

In thermal confinement, Eq. (3) holds. Eq. (9) thus becomes

$$
p(\vec{r}, t)=\left.\frac{\beta}{4 \pi C_{P}} \int d \vec{r}^{\prime} \frac{1}{\left|\vec{r}-\vec{r}^{\prime}\right|} \frac{\partial H\left(\vec{r}^{\prime}, t^{\prime}\right)}{\partial t^{\prime}}\right|_{t^{\prime}=t-\frac{\left|\vec{r}-\vec{r}^{\prime}\right|}{v_{s}}},
$$

or 
$p(\vec{r}, t)=\frac{\beta}{4 \pi C_{P}} \int d \vec{r}^{\prime} \frac{1}{\left|\vec{r}-\vec{r}^{\prime}\right|} \frac{\partial H\left(\vec{r}^{\prime}, t-\frac{\left|\vec{r}-\vec{r}^{\prime}\right|}{v_{s}}\right)}{\partial t}$,

or

$p(\vec{r}, t)=\frac{\beta}{4 \pi C_{P}} \frac{\partial}{\partial t} \int d \vec{r}^{\prime} \frac{1}{\left|\vec{r}-\vec{r}^{\prime}\right|} H\left(\vec{r}^{\prime}, t-\frac{\left|\vec{r}-\vec{r}^{\prime}\right|}{v_{s}}\right)$.

If the heating function can be decomposed into the product of spatial and temporal components, i.e., $H\left(\vec{r}^{\prime}, t^{\prime}\right)=H_{s}\left(\vec{r}^{\prime}\right) H_{t}\left(t^{\prime}\right)$,

then Eq. (12) can further be simplified to

$p(\vec{r}, t)=\frac{\beta}{4 \pi C_{P}} \frac{\partial}{\partial t} \int d \vec{r}^{\prime} \frac{H_{s}\left(\vec{r}^{\prime}\right)}{\left|\vec{r}-\vec{r}^{\prime}\right|} H_{t}\left(t-\frac{\left|\vec{r}-\vec{r}^{\prime}\right|}{v_{s}}\right)$.

The physical meaning of the Green's function has to be interpreted carefully. The spatial delta function simply represents a point absorber that provides a point acoustic source. However, because the source term of the photoacoustic equation is proportional to the first time derivative of the heating function in thermal confinement, or the second time derivative of the temperature in general, a temporal delta function in the source term can be translated into a step function in the heating function or a ramp function in the temperature rise. Therefore, the Green's function represents the response to a step heating, rather than an impulse heating, of a point absorber.

For impulse heating, i.e.,

$H_{t}\left(t^{\prime}\right)=\delta\left(t^{\prime}\right)$,

Eq. (13) can be used to compute the delta-heating response of an arbitrary absorbing object:

$p(\vec{r}, t)=\frac{\beta}{4 \pi C_{P}} \frac{\partial}{\partial t} \int d \vec{r}^{\prime} \frac{H_{s}\left(\vec{r}^{\prime}\right)}{\left|\vec{r}-\vec{r}^{\prime}\right|} \delta\left(t-\frac{\left|\vec{r}-\vec{r}^{\prime}\right|}{v_{s}}\right)$,

or

$p(\vec{r}, t)=\frac{\partial}{\partial t}\left[\frac{\beta}{4 \pi C_{P}} \frac{1}{v_{s} t} \int d \vec{r}^{\prime} H_{s}\left(\vec{r}^{\prime}\right) \delta\left(t-\frac{\left|\vec{r}-\vec{r}^{\prime}\right|}{v_{s}}\right)\right]$,

where the quantity within the square brackets is the step-heating response (the impulse response). Since the initial pressure response upon delta-heating can be expressed as

$p_{0}(\vec{r}, t)=\Gamma H_{s}\left(\vec{r}^{\prime}\right)$,

or

$p_{0}(\vec{r}, t)=\frac{v_{s}^{2} \beta}{C_{P}} H_{s}\left(\vec{r}^{\prime}\right)$,

Eq. (15) can be rewritten as

$p(\vec{r}, t)=\frac{1}{4 \pi v_{s}^{2}} \frac{\partial}{\partial t}\left[\frac{1}{v_{s} t} \int d \vec{r}^{\prime} p_{0}\left(\vec{r}^{\prime}\right) \delta\left(t-\frac{\left|\vec{r}-\vec{r}^{\prime}\right|}{v_{s}}\right)\right]$.

Equation (17) will be used to derive the responses to delta excitation of variously shaped objects. 


\section{IMAGE RECONSTRUCTION IN AN INFINITE MEDIUM}

Reconstruction for infinite acoustically homogeneous media is considered here [1]. As shown in Eq. (16), the initial photoacoustic pressure at position $\vec{r}$ excited by a $\delta(t)$ pulse equals

$p_{0}(\vec{r})=\Gamma(\vec{r}) H_{s}(\vec{r})$.

The acoustic wave $p(\vec{r}, t)$ at position $\vec{r}$ and time $t$, prompted by the initial source $p_{0}(\vec{r})$, satisfies the following wave equation:

$$
\left(\nabla^{2}-\frac{1}{v_{s}^{2}} \frac{\partial^{2}}{\partial t^{2}}\right) p(\vec{r}, t)=-\frac{p_{0}(\vec{r})}{v_{s}^{2}} \frac{d \delta(t)}{d t} .
$$

The measurement surface is represented by $S_{0}$. For the planar geometry, if another planar surface $S_{0}^{\prime}$ at infinity parallel to $S_{0}$ is added, the combination of $S_{0}^{\prime}$ and $S_{0}$ encloses the source $p_{0}\left(\vec{r}^{\prime}\right)$. For convenience, we denote $S=S_{0}+S_{0}^{\prime}$ for the planar geometry and $S=S_{0}$ for the cylindrical and spherical geometries.

The following Fourier transformation pair on variable $\bar{t}=v_{s} t$ will be used to convert the pressure between the time and frequency domains:

$$
\left\{\begin{array}{c}
\widetilde{F}(k)=\int_{-\infty}^{+\infty} F(\bar{t}) \exp (i k \bar{t}) d \bar{t} \\
F(\bar{t})=\frac{1}{2 \pi} \int_{-\infty}^{+\infty} \widetilde{F}(k) \exp (-i k \bar{t}) d k
\end{array},\right.
$$

where $k=\omega / v_{s}$ with $\omega$ being the angular frequency.

Based on Green's theorem, the spectrum $\widetilde{p}\left(\vec{r}_{0}, k\right)$ of the pressure $p\left(\vec{r}_{0}, \bar{t}\right)$ detected at $\vec{r}_{0}$ can be written as $\widetilde{p}\left(\vec{r}_{0}, k\right)=-i k \int_{V^{\prime}} d \vec{r}^{\prime} \widetilde{G}_{k}^{(o u t)}\left(\vec{r}^{\prime}, \vec{r}_{0}\right) p_{0}\left(\vec{r}^{\prime}\right)$,

where $V^{\prime}$ is the volume enclosing all of the sources $p_{0}\left(\vec{r}^{\prime}\right)$, and $\widetilde{G}_{k}^{(o u t)}\left(\vec{r}, \vec{r}_{0}\right)$ is a Green's function representing an outgoing wave:

$$
\widetilde{G}_{k}^{(o u t)}\left(\vec{r}, \vec{r}_{0}\right)=\exp \left(i k\left|\vec{r}^{\prime}-\vec{r}_{0}\right|\right) /\left(4 \pi\left|\vec{r}^{\prime}-\vec{r}_{0}\right|\right) .
$$

To reconstruct the initial photoacoustic pressure $p_{0}(\vec{r})$ from the pressure measured on $S$, we first construct a Dirichlet Green's function $\widetilde{G}_{k}^{(D)}\left(\vec{r}, \vec{r}_{1}\right)$, which satisfies the condition

$\widetilde{G}_{k}^{(D)}\left(\vec{r}, \vec{r}_{1}\right)=0$

for $\vec{r}_{1}$ on $S$ and $\vec{r}$ inside $S$. If a dipole source is chosen across $S$ for the Dirichlet Green's function, one obtains $\hat{n}_{0}^{s} \cdot \nabla_{0} \widetilde{G}_{k}^{(D)}\left(\vec{r}, \vec{r}_{0}\right)=2 \hat{n}_{0}^{s} \cdot \nabla_{0} \widetilde{G}_{k}^{(o u t)}\left(\vec{r}, \vec{r}_{0}\right)$,

where $\nabla_{0}$ denotes the gradient over variable $\vec{r}_{0}$, and $\hat{n}_{0}^{s}$ denotes the normal vector of surface $S$ pointing inward. According to Green's theorem, the acoustic pressure $\tilde{p}(\vec{r}, k)$ inside $S$ can be computed by the following surface integral:

$\tilde{p}(\vec{r}, k)=\int_{S} d S \widetilde{p}^{*}\left(\vec{r}_{0}, k\right)\left[\hat{n}_{0}^{s} \cdot \nabla_{0} \widetilde{G}_{k}^{(D)}\left(\vec{r}, \vec{r}_{0}\right)\right]$,

where * indicates a complex conjugate, which is equivalent to time reversal [2]. Since 
$p_{0}(\vec{r})=p(\vec{r}, \bar{t}=0)$,

the inverse Fourier transforming Eq. (21) leads to the following back-projection formula:

$p_{0}^{(b)}(\vec{r})=\frac{1}{\pi} \int_{-\infty}^{+\infty} d k \int_{S} d S \widetilde{p}^{*}\left(\vec{r}_{0}, k\right)\left[\hat{n}_{0}^{s} \cdot \nabla_{0} \widetilde{G}_{k}^{(o u t)}\left(\vec{r}, \vec{r}_{0}\right)\right]$.

This formula can be rewritten in the following form as the reconstructed pressure is real:

$p_{0}^{(b)}(\vec{r})=\frac{1}{\pi} \int_{S} d S \int_{-\infty}^{+\infty} d k \widetilde{p}\left(\vec{r}_{0}, k\right)\left[\hat{n}_{0}^{s} \cdot \nabla_{0} \widetilde{G}_{k}^{(i n)}\left(\vec{r}, \vec{r}_{0}\right)\right]$

where $\widetilde{G}_{k}^{(i n)}\left(\vec{r}, \vec{r}_{0}\right)$ is a Green's function corresponding to an incoming wave:

$\widetilde{G}_{k}^{(i n)}\left(\vec{r}, \vec{r}_{0}\right)=\exp \left(-i k\left|\vec{r}-\vec{r}_{0}\right|\right) /\left(4 \pi\left|\vec{r}-\vec{r}_{0}\right|\right)$.

\section{BOUNDARY CONDITIONS}

When an acoustic wave encounters an interface between two media of different acoustic impedances, the boundary conditions require that the pressure and the normal velocity (velocity normal to the interface) must be continuous. The acoustic impedance $Z$ is defined by

$Z=\rho v_{s}$.

The pressure continuity (boundary condition 1) can be considered as a consequence of the conservation of energy and the normal velocity continuity (boundary condition 2) as a consequence of the conservation of mass.

In the case that a plane wave propagating in medium 1 of impedance $Z_{1}$ is normally incident upon a planar interface with medium 2 of $Z_{2}$, the pressure reflection coefficient $r$ equals

$r=\frac{Z_{2}-Z_{1}}{Z_{2}+Z_{1}}$

Two special boundaries are of special interest in photoacoustic tomography: a soft boundary and a hard boundary.

At a soft boundary, the acoustic impedance in the incident medium is much greater than the acoustic impedance in the transmitted medium, i.e.,

$Z_{1}>Z_{2}$,

which leads to

$r=-1$.

In this case, boundary condition 1 is zero (zero pressure), which is mathematically referred to as a homogeneous Dirichlet boundary condition, and the incident pressure is reflected in equal amplitude with a sign change. An example is the acoustic wave reflection from soft tissue to air, where

$r=-0.9995$.

At a hard boundary, the acoustic impedance in the incident medium is much less than the acoustic impedance in the transmitted medium, i.e.,

$Z_{1}<<Z_{2}$,

which leads to

$r=1$.

In this case, boundary condition 2 is zero (zero normal velocity or zero normal pressure gradient), which is mathematically referred to as a homogeneous Neumann boundary condition, and the incident pressure is reflected in equal amplitude without a sign change. An example is the acoustic wave reflection from soft tissue to glass or metal. 


\section{IMAGE RECONSTRUCTION IN A FINITE MEDIUM}

When boundaries are present, the above boundary conditions must be incorporated. Here we consider a planar hard boundary in planar detection geometry (Fig. 1). One can imagine that this applies to breast imaging when the breast is compressed between a hard plate and a planar detection array. We can look at the forward problem with the boundary condition for a point source. As the problem in question is linear, the conclusion will apply to an arbitrary source.

The method of image can be used to satisfy the boundary condition. Since a hard boundary is considered, the image point source has the same sign as the original point source. If a soft boundary is considered, the image point source will have a sign that is opposite to that of the original point source. Once this image source is added, the boundary condition is satisfied; as a result, the boundary can be removed. Therefore, we have two point sources embedded in an infinite medium. As shown in Section 3, the Green's function provides the solution for a single source in an infinite medium. The solution for these two point sources in an infinite medium is a superposition (sum) of the solutions for each of the two point sources.

If we also image the detectors, we see immediately that the photoacoustic signals received by the image detectors are the same as those received by the corresponding original detectors for reasons of symmetry. Therefore, in the "imaged" infinite medium, we can use the same inverse reconstruction algorithm presented in Section 4 to reconstruct the original point source as well as the image point source. Of course, there is no need for the latter in practice. Again, this conclusion applies to an arbitrary source (photoacoustic object).

\section{Original geometry}

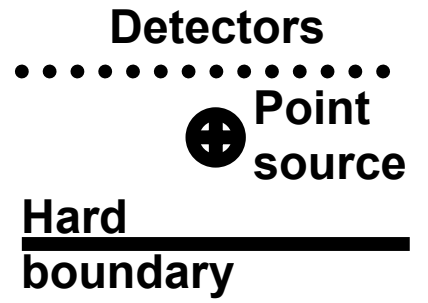

Positive images added

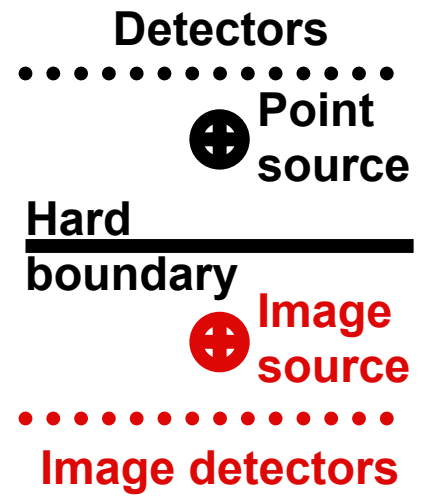

\section{Boundary removed}

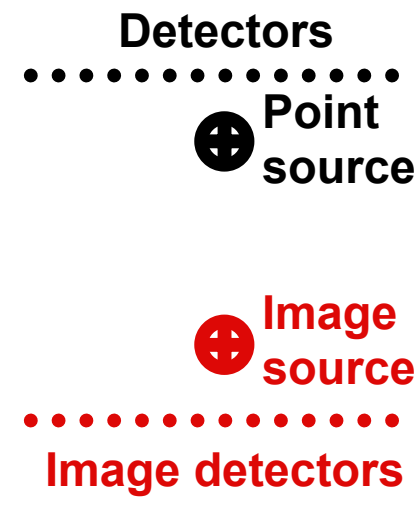

Figure 1. Inverse solution for image reconstruction when a planar hard boundary is present in photoacoustic tomography in planar detection geometry.

We can use the following sequence to reconstruct the image:

1. Image the detectors about the planar hard boundary from $\vec{r}_{0}$ to $\vec{r}_{0}^{*}$.

2. Assign the received photoacoustic signals in the original detectors to the corresponding imaged detectors with no sign change: $\tilde{p}\left(\vec{r}_{0}^{*}, k\right)=\widetilde{p}\left(\vec{r}_{0}, k\right)$. 
3. Apply the reconstruction algorithm for an infinite medium:

$$
p_{0}^{(b)}(\vec{r})=\frac{1}{\pi} \int_{-\infty}^{+\infty} d k \int_{S} d S \widetilde{p}^{*}\left(\vec{r}_{0}, k\right)\left[\hat{n}_{0}^{s} \cdot \nabla_{0} \widetilde{G}_{k}^{(o u t)}\left(\vec{r}, \vec{r}_{0}\right)\right]
$$

where $S$ includes the original and imaged detectors.

We can easily extend this algorithm to a soft boundary where a sign change is required in step 2 .

\section{SUMMARY}

In summary, the boundary conditions that should be considered in certain imaging configurations are presented and the associated inverse solutions for image reconstruction are solved. We can easily extend this algorithm to a soft boundary case where a sign change is required. We can also generalize the inverse solution to semi-cylindrical and hemispherical detection surfaces as long as the final surfaces that include both the original and imaged detection surfaces form complete cylinders or spheres.

\section{REFERENCES}

Xu MH and Wang LHV (2005). "Universal back-projection algorithm for photoacoustic computed tomography." Physical Review E 71(1): 016706.

$\mathrm{Xu}$ Y and Wang LHV (2004). "Time reversal and its application to tomography with diffracting sources." Physical Review Letters 92(3): 033902. 